\title{
Determination of the Vanadium Binding Mode on Seawater-Contacted Polyamidoxime Adsorbents
}

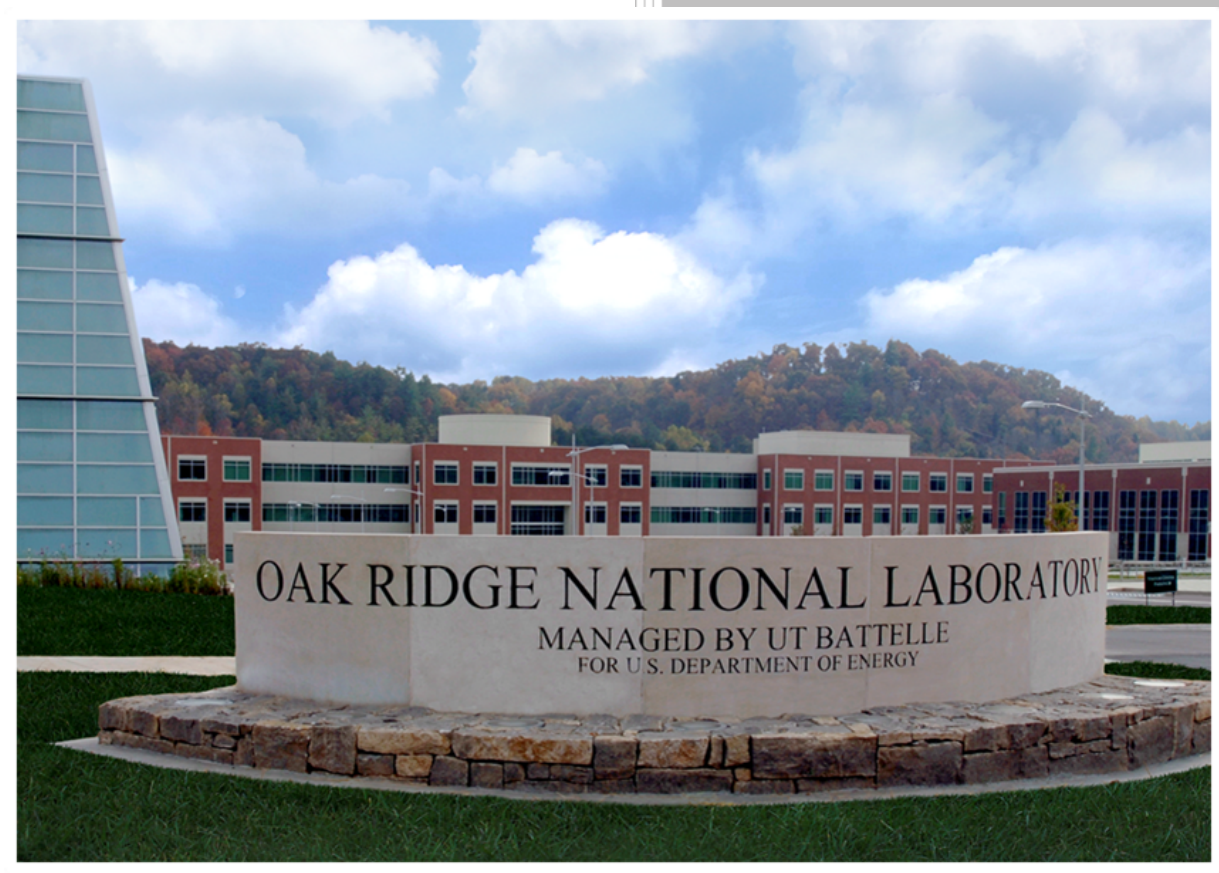

Carter W. Abney Vyacheslav Bryantsev Alexandr Ivanov Zhicheng Zhang (LBNL) Linfeng Rao (LBNL)

September 29, 2017 


\title{
DOCUMENT AVAILABILITY
}

Reports produced after January 1, 1996, are generally available free via US Department of Energy (DOE) SciTech Connect.

Website http://www.osti.gov/scitech/

Reports produced before January 1, 1996, may be purchased by members of the public from the following source:

\author{
National Technical Information Service \\ 5285 Port Royal Road \\ Springfield, VA 22161 \\ Telephone 703-605-6000 (1-800-553-6847) \\ TDD 703-487-4639 \\ Fax 703-605-6900 \\ E-mail info@ntis.gov \\ Website http://classic.ntis.gov/
}

Reports are available to DOE employees, DOE contractors, Energy Technology Data Exchange representatives, and International Nuclear Information System representatives from the following source:

Office of Scientific and Technical Information

PO Box 62

Oak Ridge, TN 37831

Telephone 865-576-8401

Fax 865-576-5728

E-mail reports@osti.gov

Website http://www.osti.gov/contact.html

This report was prepared as an account of work sponsored by an
agency of the United States Government. Neither the United States
Government nor any agency thereof, nor any of their employees, makes
any warranty, express or implied, or assumes any legal liability or
responsibility for the accuracy, completeness, or usefulness of any
information, apparatus, product, or process disclosed, or represents that
its use would not infringe privately owned rights. Reference herein to
any specific commercial product, process, or service by trade name,
trademark, manufacturer, or otherwise, does not necessarily constitute
or imply its endorsement, recommendation, or favoring by the United
States Government or any agency thereof. The views and opinions of
authors expressed herein do not necessarily state or reflect those of the
United States Government or any agency thereof.


Chemical Sciences Division

\title{
DETERMINATION OF THE VANADIUM BINDING MODE ON SEAWATER-
} CONTACTED POLYAMIDOXIME ADSORBENTS

\author{
Carter W. Abney \\ Vyacheslav Bryantsev \\ Alexandr Ivanov \\ Zhicheng Zhang (LBNL) \\ Linfeng Rao (LBNL)
}

Date Published:

September 29, 2017

Prepared by

OAK RIDGE NATIONAL LABORATORY

Oak Ridge, TN 37831-6283

managed by

UT-BATTELLE, LLC

for the

US DEPARTMENT OF ENERGY

under contract DE-AC05-00OR22725 



\section{CONTENTS}

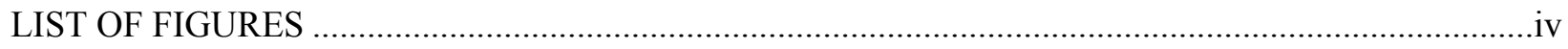

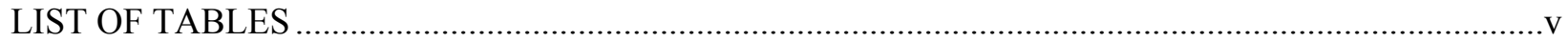

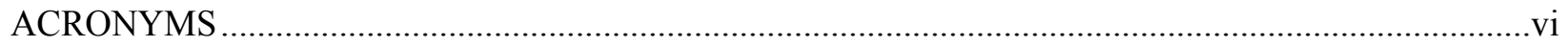

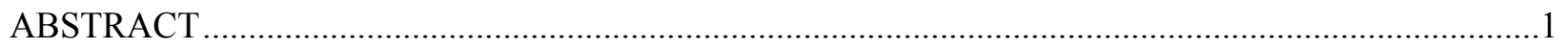

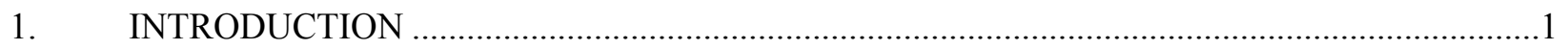

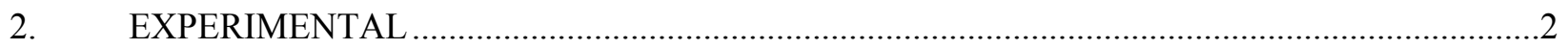

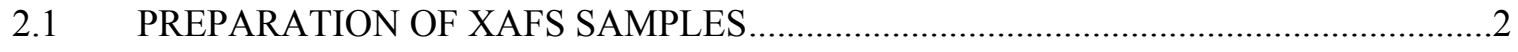

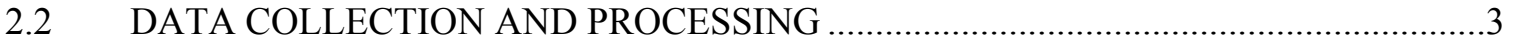

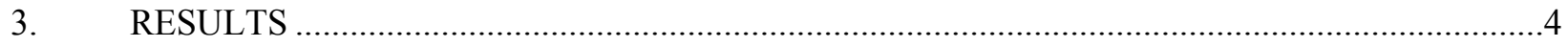

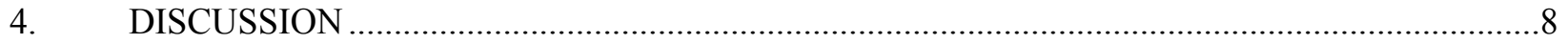

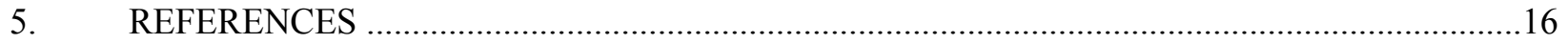




\section{LIST OF FIGURES}

Figure 1. X-ray Absorption Near Edge Spectra (XANES) for representative brine- and seawatercontacted adsorbents, $\mathrm{Na}\left[\mathrm{V}(\mathrm{IDO})_{2}\right.$ ] single crystal, and mineral standards of $\mathrm{Na}_{3} \mathrm{VO}_{4}, \mathrm{~V}_{2} \mathrm{O}_{4}$, and $\mathrm{V}_{2} \mathrm{O}_{3}$ displaying $\mathrm{E}_{0}$ values for oxidation states of $\mathrm{V}$, IV, and III, respectively......

Figure 2. EXAFS spectra (left) and magnitude of the Fourier transform (right) of small molecule standards and seawater-contacted adsorbents.

Figure 3. Representative EXAFS spectra for seawater contacted adsorbents and simulated scattering paths for computationally proposed vanadium (IV) and vanadium (V) binding modes. 


\section{LIST OF TABLES}

Table 1. Concentration of metals in field test seawater and as extracted by polymer adsorbents analyzed by XAFS.

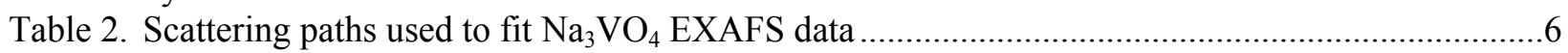

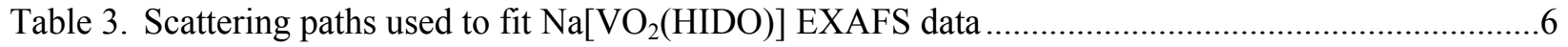

Table 4. Scattering paths used to fit $\mathrm{Na}\left[\mathrm{V}(\mathrm{IDO})_{2}\right]$ EXAFS data............................................................

Table 5. Scattering paths used to fit EXAFS data of seawater-contacted adsorbents .................................

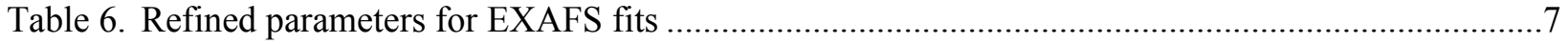

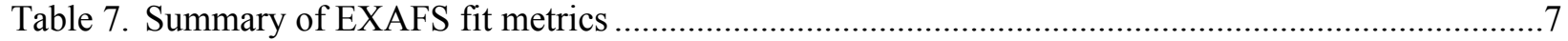




\section{ACRONYMS}

AF-1 ORNL adsorbent composed of poly(amidoxime- $c o$-itaconic acid)

AI-8

APS

DFT

ORNL adsorbent composed of poly(amidoxime-co-vinyl phosphonic acid)

EXAFS

$\mathrm{H}_{3}$ IDO

ORNL

PNNL

ppm

Advanced Photon Source

Density Functional Theory

Extended X-ray Absorption Fine Structure

Cyclic Imidedioxime

Oak Ridge National Laboratory

Pacific Northwest National Laboratory

SSRL

XAFS

Stanford Synchrotron Radiation Lightsource

XANES X-ray Absorption Near Edge Structure 


\begin{abstract}
Adsorbents developed for the recovery of uranium from seawater display poor selectivity over other transition metals present in the ocean, with vanadium particularly problematic. To improve selectivity, an indispensable step is the positive identification of metal binding environments following actual seawater deployment. In this work we apply x-ray absorption fine structure (XAFS) spectroscopy to directly investigate the vanadium binding environment on seawater-deployed polyamidoxime adsorbents. Comparison of the x-ray absorption near edge spectra (XANES) reveal marked similarities to a recently reported non-oxido vanadium (V) structure formed upon binding with cyclic imidedioxime, a byproduct of generating amidoxime functionalities. Density functional theory (DFT) calculations provided a series of putative vanadium binding environments for both vanadium (IV) and vanadium (V) oxidation states, and with both amidoxime and cyclic imidedioxime. Fits of the extended XAFS (EXAFS) data confirmed vanadium $(\mathrm{V})$ is bound exclusively by the cyclic imidedioxime moiety in a 1:2 metal:ligand fashion, though a modest structural distortion is also observed compared to crystal structure data and computationally optimized geometries which is attributed to morphology effects from the polymer graft chain and the absence of crystal packing interactions. These results demonstrate that improved selectivity for uranium over vanadium can be achieved by suppressing the formation of cyclic imidedioxime during preparation of polyamidoxime adsorbents for seawater uranium recovery.
\end{abstract}

\title{
1. INTRODUCTION
}

The recovery of uranium from seawater provides an effectively limitless unconventional reserve for ensuring long-term fuel availability for nuclear power generation (Kim, Tsouris et al. 2013). The most technologically mature adsorbents, initially reported in the early 1980's, are prepared by graft polymerization of polyacrylonitrile and a hydrophilic co-monomer from the surface of a robust polymer trunk (Tamada 2010). Subsequent treatment with hydroxylamine generates the uranium-binding polyamidoxime functionality. Current state-of-the-art adsorbents developed at Oak Ridge National Laboratory (ORNL) have achieved improvements in uranium adsorption performance, exceeding the previous generation of adsorbents developed by the Japan Atomic Energy Agency by more than threefold (Kim, Tsouris et al. 2013; Brown, Yue et al. 2016; Das, Brown et al. 2016; Das, Oyola et al. 2016a; Das, Oyola et al. 2016b).

While already capable of providing a financial backstop (Lindner and Schneider 2015), suppressing the potential for dramatic fluctuations in uranium market price, further reductions in cost are necessary for seawater uranium recovery to be financially viable. Improvements in selective binding of uranium over other transition metals present in seawater is an avenue by which this can be achieved (Flicker Byers and Schneider 2015). Additionally, while uranium is not known to participate in any biological processes, the transition metals which are co-extracted by ORNL adsorbents are important to marine ecology (Morel and Price 2003). Large-scale deployment of the current uranium adsorbents would also result in severe depletion regions of vanadium, copper, nickel, iron, and zinc, with severe potential ramifications. Thus, improvements in adsorbent selectivity are critical with respect to uranium recovery efficiency, financial economy, and ocean ecology.

Achieving selectivity for uranium binding, and thus recovery, requires an understanding of the binding environments for all metals extracted by the adsorbents following prolonged contact in environmental seawater. Due to adsorbing numerous metal species, possessing no significant long-range atomic structure, and a relatively low concentration of metals, traditional spectroscopic and x-ray scattering approaches have been ineffective in elucidating how uranium (and other metals) are bound by the polyamidoxime adsorbents. Extensive efforts involving small molecule surrogates and computational approaches have thus been pursued in an attempt to approximate the binding occurring on the adsorbent 
polymer (Kang, Vukovic et al. 2012; Tian, Teat et al. 2012; Vukovic, Watson et al. 2012; Tian, Teat et al. 2013). However, polymers are significantly more complex than isolated small molecules (Dobrynin and Rubinstein 2005), and there is no assurance these results are indeed representative of adsorbent binding behavior.

In contrast to more routine characterization techniques, X-ray absorption fine structure (XAFS) spectroscopy relies on the absorption of x-rays possessing characteristic energies, exciting core electrons from the element being investigated. Due to elements possessing different orbital energies, XAFS is an element-discriminating technique capable of interrogating only the specific element of interest, typically with no spectral complications from other elements present in the sample. It has no requirements regarding chemical ordering or sample phase, and advanced detection methods allow investigation of metals present at part-per-million (ppm) concentrations.

Recently we reported the application of x-ray absorption fine structure (XAFS) spectroscopy for the investigation of the uranium coordination environment on a poly(amidoxime-co-itaconic acid) adsorbent (AF-1) prepared at ORNL (Abney, Mayes et al. 2016). In addition to being the first instance XAFS was applied to investigate seawater-deployed adsorbents, it was also the first instance the uranium binding mode was directly interrogated successfully. In this study we observed that no previously reported crystal structures or computationally predicted binding modes were adequately able to represent the uranium binding environment, as revealed by fitting the extended XAFS (EXAFS) region of the spectrum, and an unanticipated binding mode involving a $\mu^{2}$-oxo-bridged transition metal was proposed. We reported similar findings for a related poly(amidoxime-co-vinyl phosphonic acid) adsorbent (AI-8) as well (Abney, Das et al. 2016).

Although uranium is the desired metal to be recovered, vanadium is the transition metal which the polymer adsorbents bind most readily. Assuming both vanadium and uranium compete for the same binding sites, substitution of uranium for vanadium would potentially achieve up to a $50 \times$ improvement in uranium uptake. Previous computational studies predicted neither vanadium (IV) nor vanadium (V) have significant affinity for the amidoxime functionality (Ivanov and Bryantsev 2016; Mehio, Ivanov et al. 2016), while small molecule studies have revealed cyclic imidedioxime ( $\left.\mathrm{H}_{3} \mathrm{IDO}\right)$, a thermodynamicallyfavored byproduct formed during treatment of adjacent nitriles with hydroxylamine (Eloy and Lenaers 1962; Astheimer, Schenk et al. 1983; Katritzky, Huang et al. 2012) will form a highly stable and rare nonoxido vanadium (V) complex (Leggett, Parker et al. 2016).

In this work we apply XAFS to positively identify the vanadium binding environment for both aforementioned polymer adsorbents, leveraging DFT-optimized geometries to afford structure models of vanadium $(\mathrm{IV} / \mathrm{V})$ coordination at different potential metal binding sites. Importantly, representative small molecule crystal structures are not available to depict many of the proposed binding modes, and computational approaches thus afford a relatively quick and inexpensive means by which accurate metal geometries can be screened. Moreover, through application of thermochemical calculations and, when available, comparison with small molecule crystal structures, this approach also affords a means by which structural paradigms generated through investigation of small molecule complexes can be validated for complex environmental samples.

\section{EXPERIMENTAL}

\subsection{PREPARATION OF XAFS SAMPLES}

Samples investigated by XAFS were prepared from adsorbents of AF-1 (Das, Oyola et al. 2016a) and AI8 (Das, Oyola et al. 2016b) formulations. The adsorbents were first conditioned with $\mathrm{KOH}$ and either 
contacted for 56 days at the Marine Sciences Laboratory of Pacific Northwest National Laboratory (PNNL), as reported in previous publications (Das, Oyola et al. 2016a; Das, Oyola et al. 2016b; Gill, Kuo et al. 2016), or in a brine solution containing 6 ppm uranyl nitrate and $3.4 \mathrm{ppm}$ sodium orthovanadate (Abney, Mayes et al. 2016). Metal uptake for seawater-deployed adsorbents is reported below in Table 1.

Table 1. Concentration of metals in field test seawater and as extracted by polymer adsorbents analyzed by XAFS

\begin{tabular}{cccc}
\hline Metal & $\begin{array}{c}\text { Seawater } \\
(\boldsymbol{\mu g} / \mathbf{~ k g})\end{array}$ & $\begin{array}{c}\text { AF-1 } \\
(\mathbf{g ~ M} / \mathbf{k g - a d s} .)\end{array}$ & $\begin{array}{c}\text { AI-8 } \\
(\mathbf{g ~ M} / \mathbf{k g - a d s} .)\end{array}$ \\
\hline $\mathrm{V}$ & 1.48 & 5.8 & 7.0 \\
$\mathrm{U}$ & 2.84 & 3.1 & 2.8 \\
$\mathrm{Fe}$ & 2.20 & 0.8 & 4.0 \\
$\mathrm{Cu}$ & 0.54 & 0.7 & 0.5 \\
$\mathrm{Ni}$ & 0.56 & 0.4 & 0.4 \\
$\mathrm{Zn}$ & 2.10 & 0.9 & 0.6 \\
$\mathrm{Sr}$ & --- & 0.1 & $<0.1$ \\
$\mathrm{Cr}$ & 0.18 & $<0.1$ & $<0.1$ \\
$\mathrm{Mn}$ & 1.20 & $<0.1$ & 0.9 \\
\hline
\end{tabular}

The mass of uranium needed to achieve a $1-2.5$ absorption length edge step was calculated for each small molecule standard based on the elemental composition and mass absorption coefficient for each element. $\mathrm{Na}\left[\mathrm{VO}_{2}(\mathrm{HIDO})\right], \mathrm{Na}\left[\mathrm{V}(\mathrm{IDO})_{2}\right]$, and vanadium oxide standards were prepared as reported previously (Leggett, Parker et al. 2016), ground with an agate mortar and pestle, and blended with boron nitride to the appropriate concentration. For seawater contacted fibers, approximately $100 \mathrm{mg}$ wet fibers were washed with DI water, dried overnight at $40{ }^{\circ} \mathrm{C}$ in a vacuum oven, immersed in liquid nitrogen and pulverized with a mortar and pestle. Samples contacted with the brine solution were diluted with D-(+)Glucose to minimize self-absorption effects, while samples contacted with seawater were not diluted.

Small molecule and vanadium oxide standards were loaded on an aluminum holder with rectangular opening of $20 \mathrm{~mm}(l) \times 2 \mathrm{~mm}(w)$ and a thickness of $0.5-1.0 \mathrm{~mm}$, sealed with Kapton tape. Polymer samples were enclosed in a Nylon washer of $4.953 \mathrm{~mm}$ inner diameter, pressed into a self-supporting pellet, and sealed on both sides with Kapton tape. The entire sample was then placed into a baggie formed of Kapton film, which was then sealed with Kapton tape. This method of sample containment was approved by the Advanced Photon Source (APS) Radiation Safety Review Board to ensure double containment for analysis of radioactive samples, as seawater-contacted adsorbents also contain appreciable quantities of natural uranium.

\subsection{DATA COLLECTION AND PROCESSING}

XAFS data were collected at the V K-edge (5465 eV) on beamline 10-BM-B of the APS (Kropf, Katsoudas et al. 2010) for the polymers and vanadium oxide standards, and beamline 4-3 of the Stanford Synchrotron Radiation Lightsource (SSRL) for $\mathrm{Na}\left[\mathrm{VO}_{2}(\mathrm{HIDO})\right]$ and $\mathrm{Na}\left[\mathrm{V}(\mathrm{IDO})_{2}\right.$ ] small molecule standards. XAFS spectra of the vanadium oxides and small molecule standards were collected in transmission mode, while polymer samples were collected using a fluorescence detector. For APS beamline 10-BM-B, a Hitachi Vortex-ME4 four-element silicon drift fluorescence detector was used. For SSRL beamline 4-3, a Lytle or Vortex detector was used. For all samples, data sets were collected until 
adequate signal to noise was obtained, in all instances between 3 and 10 scans. The x-ray white beam was monochromatized by a $\mathrm{Si}(111)$ monochromator and detuned by $50 \%$ to reduce the contribution of higher-order harmonics. The K-edge of a vanadium foil was used as the reference for energy calibration and measured simultaneously for all samples. All samples were collected at room temperature.

Data were processed with the Athena and Artemis programs of the IFEFFIT package(Ravel and Newville 2005). Reference foil data were aligned to the first zero-crossing of the second derivative of normalized $\mu(\mathrm{E})$ data, which was calibrated to the literature $\mathrm{E}_{0}$ value for the vanadium K-edge. Spectra were averaged in $\mu(E)$ prior to normalization. Background removal was achieved by spline fitting. EXAFS

data were extracted above the threshold energy, $E_{0}$. FEFF 9 (Rehr and Albers 2000) was used to calculate theoretical phases and amplitudes from structure models consisting of crystal structures or the Cartesian coordinates of geometrically optimized computational models. All data were initially fitted with simultaneous k-weighting of 1,2, and 3, then finalized with $\mathrm{k}^{2}$-weighting in R-space. Fit windows in kspace were determined based on the lowest quality data collected, and for all data sets were from $2.75-$ $10.2 \AA^{-1}$. Fit windows in R-space were determined on a case-by-case basis, based on the features apparent in the spectrum. In all fits, the amplitude reduction factor $\left(\mathrm{S}_{0}{ }^{2}\right)$ and energy shift of the photoelectron $\left(\Delta \mathrm{E}_{0}\right)$ were global parameters. Independent structural parameters determined by the fits included the change in the scattering half path length $\left(\Delta \mathrm{R}_{i}\right)$ and the relative mean square displacement of the scattering element $\left(\sigma_{i}^{2}\right)$. For each fit, the number of variables was not permitted to exceed $2 / 3$ the number of independent points, in keeping with the Nyquist criterion (Calvin 2013).

\section{RESULTS}

Comparison of the X-ray absorption near edge (XANES) spectra for the seawater-contacted adsorbents, vanadium oxide standards, and $\mathrm{Na}\left[\mathrm{V}(\mathrm{IDO})_{2}\right]$ is particularly revealing with regards to the vanadium coordination environment and binding mode (Figure 1). A common pre-edge feature at $5470 \mathrm{eV}$ is apparent for both seawater-contacted adsorbents and $\mathrm{Na}\left[\mathrm{V}(\mathrm{IDO})_{2}\right]$. This feature is attributable to the $1 \mathrm{~s} \rightarrow 3 \mathrm{~d}$ transition arising from the distorted octahedral $\mathrm{V}$ coordination environment, identified computationally as due to a Pseudo Jahn-Teller effect. Comparison of the first derivative of the absorption edge reveals the absorption edge, $\mathrm{E}_{0}$, for these samples to be located at $5482 \mathrm{eV}$. A similar $\mathrm{E}_{0}$ is identified in the $\mathrm{V}(\mathrm{V})$ metal oxide standard, $\mathrm{Na}_{3} \mathrm{VO}_{4}$, particularly when compared to $\mathrm{V}(\mathrm{IV})$ and $\mathrm{V}(\mathrm{III})$ species. Although anticipated from the literature and previous computational work (Baes and Mesmer 1976; Mehio, Johnson et al. 2015; Ivanov and Bryantsev 2016; Mehio, Ivanov et al. 2016), this is the first instance the +5 oxidation state is experimentally confirmed for adsorbent-bound $\mathrm{V}$. 


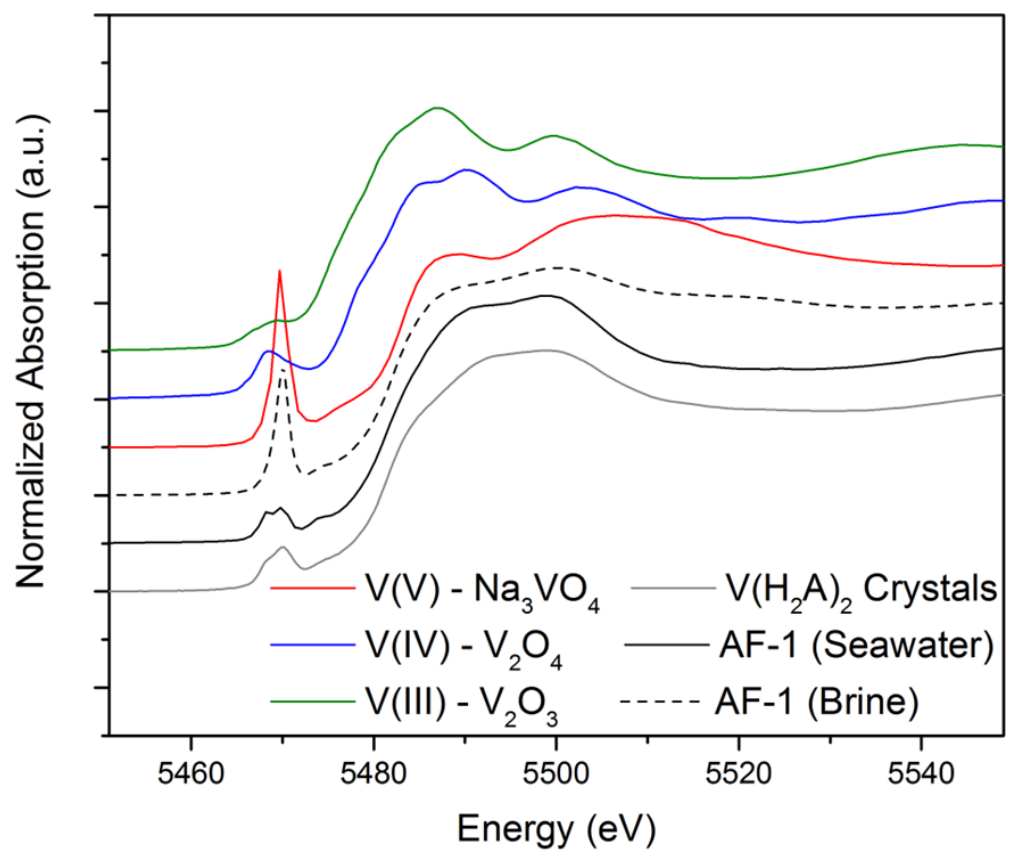

Figure 1. X-ray Absorption Near Edge Spectra (XANES) for representative brine- and seawater-contacted adsorbents, $\mathrm{Na}\left[\mathrm{V}(\mathrm{IDO})_{2}\right]$ single crystal, and mineral standards of $\mathrm{Na}_{3} \mathrm{VO}_{4}, \mathrm{~V}_{2} \mathrm{O}_{4}$, and $\mathrm{V}_{2} \mathrm{O}_{3}$ displaying $\mathrm{E}_{0}$ values for oxidation states of $\mathrm{V}, \mathbf{I V}$, and III, respectively. The absorption edge of the brine- and seawatercontacted adsorbents possess an $\mathrm{E}_{0}$ value consistent with $\mathrm{Na}_{3} \mathrm{VO}_{4}(5482 \mathrm{eV})$, supporting an oxidation state of $\mathrm{V}(\mathrm{V})$. $\mathrm{E}_{0}$ values for $\mathrm{V}(\mathrm{IV})$ and $\mathrm{V}(\mathrm{III})$ are $5478 \mathrm{eV}$ and $5475 \mathrm{eV}$, respectively.

Although XANES analysis provides preliminary support for the small molecule determined binding mode, fits of the extended XAFS (EXAFS) region afford greater rigor and determination of a more precise $\mathrm{V}$-binding environment (Figure 2). Fits of $\mathrm{Na}_{2}\left[\mathrm{VO}_{2}\right.$ (IDO)] and $\mathrm{Na}\left[\mathrm{V}(\mathrm{IDO})_{2}\right]$ were readily achieved using scattering paths calculated from the corresponding crystal structures (Leggett, Parker et al. 2016), as displayed in Figure 2. Nevertheless, for the seawater-contacted adsorbents the scattering paths from $\mathrm{Na}\left[\mathrm{V}(\mathrm{IDO})_{2}\right]$ required elongation of approximately $0.1 \AA$ in order to cleanly superposition the experimental features observed in the data; a fit of the data was then obtained readily, achieving good statistics and reasonable physical parameters. This modest distortion was not observed in the crystal structure, geometrically-optimized computational model, and is proposed to be attributable to morphology effects associated with greater steric encumbrance arising from inclusion of the $\mathrm{H}_{3}$ IDO species within the polymer. The influence of graft chain morphology was previously proposed to be a significant factor in both adsorbent performance (Saito, Brown et al. 2014) and metal binding mode for U (Abney, Das et al. 2016; Abney, Mayes et al. 2016), which are now further substantiated by the EXAFS results for V binding discussed above. Fit parameters for all data sets are provided in Tables 2-7. 

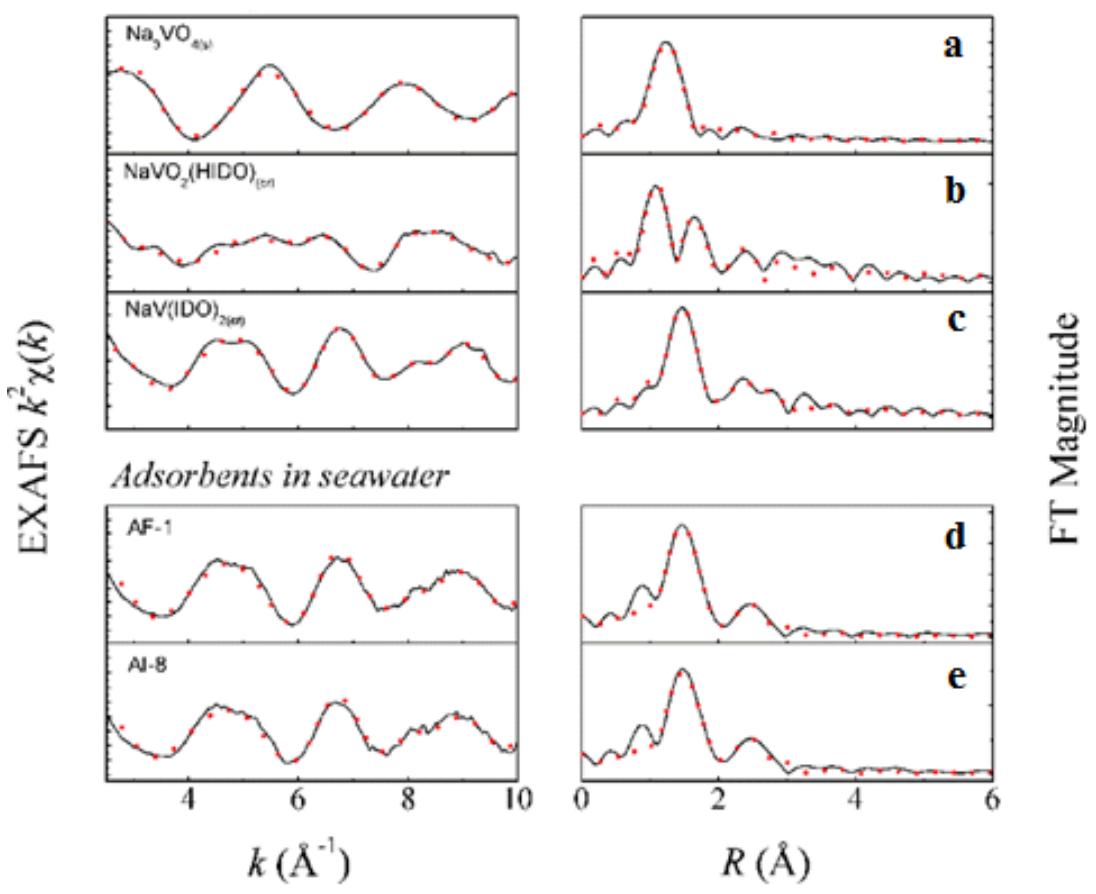

Figure 2. EXAFS spectra (left) and magnitude of the Fourier transform (right) of small molecule standards and seawater-contacted adsorbents. (Upper) Small molecule standards: a, $\mathrm{Na}_{3} \mathrm{VO}_{4(\mathrm{~s})} ; \mathbf{b} \mathrm{Na}\left[\mathrm{VO}_{2}(\mathrm{HIDO})\right]_{(\mathrm{cr})} ; \mathbf{c}$ $\mathrm{Na}\left[\mathrm{V}(\mathrm{IDO})_{2}\right]_{(\mathrm{cr})}$. (Lower) Seawater-contacted adsorbents: d AF-1; e AI-8. All samples show the data as a solid black line and the fit as a dotted red line.

Table 2. Scattering paths used to fit $\mathrm{Na}_{3} \mathrm{VO}_{4}$ EXAFS data

\begin{tabular}{ccc}
\hline Path & Degeneracy & Initial Distance $(\AA)$ \\
\hline $\mathrm{V} \rightarrow \mathrm{O}$ & 4 & 1.691 \\
$\mathrm{~V} \rightarrow \mathrm{O} \rightarrow \mathrm{O}$ & 12 & 3.072
\end{tabular}

Table 3. Scattering paths used to fit $\mathrm{Na}\left[\mathrm{VO}_{2}(\mathrm{HIDO})\right]$ EXAFS data

\begin{tabular}{ccc}
\hline \multicolumn{1}{c}{ Path } & Degeneracy & Initial Distance $(\AA)$ \\
\hline $\mathrm{V} \rightarrow \mathrm{O}$ & 2 & 1.637 \\
$\mathrm{~V} \rightarrow \mathrm{N}_{\text {imide }}$ & $3^{a}$ & 1.989 \\
$\mathrm{~V} \rightarrow \mathrm{N}_{\text {oxime }}$ & $4^{b}$ & 2.898 \\
$\mathrm{~V} \rightarrow \mathrm{O}_{\text {oxime }} \rightarrow \mathrm{N}_{\text {oxime }}$ & 8 & 3.107
\end{tabular}

${ }^{a}$ Including $\mathrm{V} \rightarrow \mathrm{N}_{\text {imide }}$ (degeneracy: 1) and $\mathrm{V} \rightarrow \mathrm{O}_{\text {oxime }}$ (degeneracy: 2)

${ }^{b}$ Including $\mathrm{V} \rightarrow \mathrm{N}_{\text {oxime }}$ (degeneracy: 2) and $\mathrm{V} \rightarrow \mathrm{C}_{\text {cycle }}$ (degeneracy: 2) 
Table 4. Scattering paths used to fit $\mathrm{Na}\left[\mathrm{V}(\mathrm{IDO})_{2}\right]$ EXAFS data

\begin{tabular}{ccc}
\hline \multicolumn{1}{c}{ Path } & Degeneracy & Initial Distance $(\AA)$ \\
\hline $\mathrm{V} \rightarrow \mathrm{O}_{\text {oxime }}$ & $6^{a}$ & $1.902 / 1.946$ \\
$\mathrm{~V} \rightarrow \mathrm{N}_{\text {oxime }}$ & 8 & $2.891 / 2.999$ \\
$\mathrm{~V} \rightarrow \mathrm{N}_{\text {oxime }} \rightarrow \mathrm{O}_{\text {oxime }}$ & 16 & $3.105 / 3.167$ \\
${ }^{a}$ Including $\mathrm{V} \rightarrow \mathrm{N}_{\text {imide }}$ (degeneracy: 2) and $\mathrm{V} \rightarrow \mathrm{O}_{\text {oxime }}$ (degeneracy: 4)
\end{tabular}

Table 5. Scattering paths used to fit EXAFS data of seawater-contacted adsorbents

\begin{tabular}{ccc}
\hline \multicolumn{1}{c}{ Path } & Degeneracy & Initial Distance $(\AA)$ \\
\hline $\mathrm{V} \rightarrow \mathrm{O}_{\text {oxime }}$ & 4 & 1.946 \\
$\mathrm{~V} \rightarrow \mathrm{N}_{\text {imide }}$ & 2 & 2.157 \\
$\mathrm{~V} \rightarrow \mathrm{N}_{\text {oxime }}$ & 8 & 2.999 \\
$\mathrm{~V} \rightarrow \mathrm{N}_{\text {oxime }} \rightarrow \mathrm{O}_{\text {oxime }}$ & 16 & 3.167
\end{tabular}

Note: The structure model for seawater-contacted adsorbents was lengthened from the crystal structure to account for polymer morphology influences.

Table 6. Refined parameters for EXAFS fits

\begin{tabular}{ccccc}
\hline Parameter & $\mathbf{N a}\left[\mathbf{V O}_{2}(\mathbf{H I D O})\right]$ & $\mathbf{N a}\left[\mathbf{V}(\mathbf{I D O})_{2}\right]$ & $\mathbf{A F - 1}$ & $\mathbf{A I - 8}$ \\
\hline $\mathrm{S}_{0}{ }^{2}$ & $0.98 \pm 0.20$ & $0.72 \pm 0.15$ & $1.01 \pm 0.07$ & $0.83 \pm 0.07$ \\
$\Delta \mathrm{E}_{0}(\mathrm{eV})$ & $-2.5 \pm 3.0$ & $7.8 \pm 4.3$ & $-1 \pm 2$ & $0 \pm 2$ \\
$\Delta \mathrm{r}_{\mathrm{O}(\text { oxime })}(\AA)$ & $0.03 \pm 0.04$ & $0.05 \pm 0.02$ & $-0.02 \pm 0.01$ & $-0.01 \pm 0.01$ \\
$\sigma_{\mathrm{O}}^{2}\left(\times 10^{-3} \AA^{2}\right)$ & $3.5 \pm 0.9$ & $2.1 \pm 0.9$ & $2.0 \pm 0.4$ & $2.0 \pm 0.4$ \\
$\Delta \mathrm{r}_{\mathrm{N} \text { (imide) }}(\AA)$ & $0.003 \pm 0.03$ & --- & $0.21 \pm 0.02$ & $0.23 \pm 0.03$ \\
$\sigma_{\mathrm{N}}^{2}\left(\times 10^{-3} \AA^{2}\right)$ & $4.5 \pm 1.0$ & $2.9 \pm 0.8$ & $5 \pm 2$ & $4 \pm 2$ \\
$\Delta \mathrm{r}_{\mathrm{N}(\text { oxime })}(\AA)$ & $0.01 \pm 0.05$ & $0.02 \pm 0.02$ & $-0.12 \pm 0.01$ & $-0.11 \pm 0.2$
\end{tabular}

Table 7. Summary of EXAFS fit metrics

\begin{tabular}{ccccc}
\hline & $\mathbf{N a}\left[\mathbf{V O} \mathbf{O}_{2}(\mathbf{H I D O})\right]$ & $\mathbf{N a}\left[\mathbf{V}(\mathbf{I D O})_{2}\right]$ & $\mathbf{A F - 1}$ & $\mathbf{A I - 8}$ \\
\hline k-range & $2.5-10.2$ & $2.75-10.2$ & $2.75-10.2$ & $2.75-10.2$ \\
R-range & $1.0-3.5$ & $1.1-3.5$ & $1.1-3.5$ & $1.1-3.5$ \\
$\mathrm{~N}_{\text {IDP }}$ & 11.9 & 11.3 & 11.3 & 11.3 \\
$\mathrm{~N}_{\text {var }}$ & 7 & 6 & 7 & 7 \\
$\chi_{v}{ }^{2}$ & 586 & 653 & 88 & 71 \\
$\mathrm{R}$ & $4.5 \%$ & $1.5 \%$ & $1.3 \%$ & $1.7 \%$
\end{tabular}


To eliminate the possibility of other potential V-binding modes contributing significantly to the EXAFS spectra for the seawater-contacted adsorbents, structure models were generated from the computationally optimized structures displaying alternative binding motifs (Figure 3). Calculation of the theoretical scattering paths from the atoms in the first coordination sphere was performed using FEFF 9 (Rehr and Albers 2000; Rehr, Kas et al. 2010); all potential binding modes including a vanadium oxide or hydroxide species possess an intense scattering path at low radial distance which is not present in the spectra collected for seawater contacted adsorbents. The combination of a good quality fit from the $\mathrm{Na}\left[\mathrm{V}(\mathrm{IDO})_{2}\right]$ structure model, combined with the negative results obtained from alternate models afforded by computational study definitively confirms the V-binding mode on the seawater-contacted adsorbents is consistent with the small molecule behavior identified by thermochemical titrations and computational investigation.

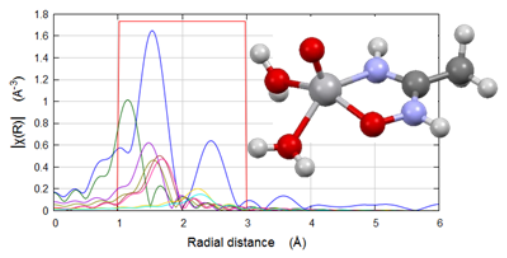

V(IV) 1:1 Acyclic

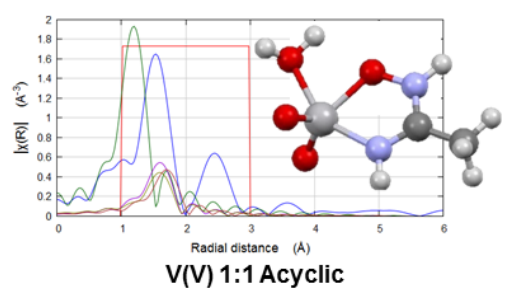

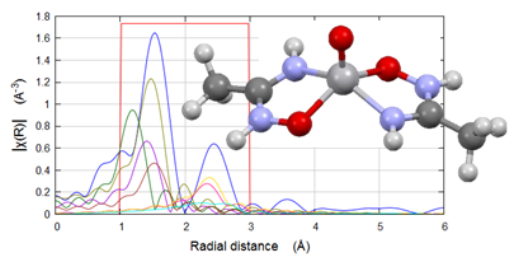

V(IV) 2:1 Acyclic

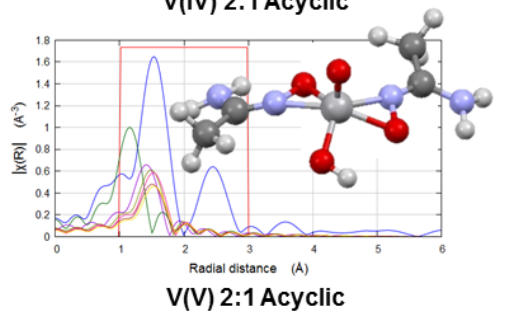

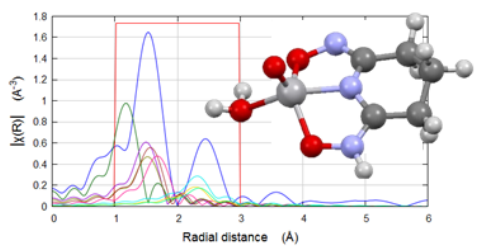

V(IV) 1:1 Cyclic

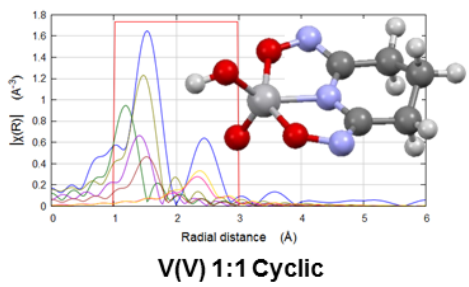

Figure 3. Representative EXAFS spectra for seawater contacted adsorbents and simulated scattering paths for computationally proposed vanadium (IV) and vanadium (V) binding modes. (Upper) Geometrically optimized vanadium (IV) binding modes with amidoxime and cyclic imidedioxime. (Lower) Geometrically optimized vanadium $(\mathrm{V})$ binding modes with amidoxime and cyclic imidedioxime. Experimental EXAFS data are displayed in blue with simulated scattering paths superpositioned in various colors. The red box displays the fit window.

\section{DISCUSSION}

In this work we determined the vanadium binding mode for two amidoxime-derived polymeric adsorbents through application of DFT calculation and XAFS spectroscopy. Previous computational and small molecule studies suggested vanadium is weakly bound by amidoxime for both vanadium (IV) and (V), while the formation of a strong non-oxido vanadium (V) species was expected upon binding with the cyclic imidedioxime byproduct (Mehio, Johnson et al. 2015; Ivanov and Bryantsev 2016; Leggett, Parker et al. 2016; Mehio, Ivanov et al. 2016). Nevertheless, any inference from such work of vanadium binding by actual polyamidoxime adsorbents is inherently predicated on the assumption that the graft polymers behave analogously to their monomeric precursors - a paradigm contested by recent EXAFS investigations of uranyl as bound by the same adsorbent morphologies investigated in this work. Polymer morphology effects attributable to graft chain density, polydispersity, and solvophilicity have all been documented (Dobrynin and Rubinstein 2005), and seawater constitutes a highly complex and variable matrix replete with fluctuations in metal concentration, dissolved organic content, salinity, $\mathrm{pH}$, temperature, and biological activity. Accordingly, direct validation of the vanadium binding environment on representative seawater-contacted adsorbents is indispensable for achieving any definitive conclusions. 
To validate the theoretical and experimental studies in aqueous solution and confirm the extension of small molecule data to bulk materials, seawater-contacted amidoxime-functionalized adsorbents were analyzed by EXAFS, revealing a vanadium (V) coordination environment composed of two imidedioxime functionalities and confirming the oxidation state of adsorbent-bound vanadium. Notably, while a vanadium $(\mathrm{V})$ oxidation state is anticipated to predominate under environmental conditions (Baes and Mesmer 1976), the possibility of vanadium (IV) binding has been proposed due to generation through biologic activity and greater abundance in the less oxidizing environment beneath the phototropic zone.

From these results we can also conclude the imide-dioxime form is solely responsible for the extremely strong sorption of vanadium by poly(acrylamidoxime) sorbents. Suppression of this functional group will thus result in less vanadium uptake and afford greater selectivity for uranium. Furthermore, the much higher stability of the non-oxido vanadium (V) complex than the uranyl complex with cyclic imidedioxime (Tian, Teat et al. 2012; Tian, Teat et al. 2013; Endrizzi, Melchior et al. 2015; Leggett, Parker et al. 2016), as well as the wide $\mathrm{pH}$ region where the vanadium (V) complex is stable also explain the difficulty removing vanadium from the amidoxime-based sorbents during the elution process, even under highly acidic conditions (Pan, Kuo et al. 2015). Another important corollary is that the itaconic acid or vinyl phosphonic acid co-polymerized with acrylonitrile do not play a direct role in vanadium binding, as evidenced by indistinguishable binding environments on both seawater-deployed adsorbents. While improvements in uranium adsorption as a function of comonomer incorporation have been documented (Das, Oyola et al. 2016a; Das, Oyola et al. 2016b), it is likely their role is to increase polymer hydrophilicity or change the graft chain morphology (Saito, Brown et al. 2014).

Of equal importance, these results may also partially rationalize previously reported EXAFS data that reveal no cyclic imide-dioxime contribution to the uranium coordination environment (Abney, Das et al. 2016; Abney, Mayes et al. 2016). Due to weaker binding, it is possible that uranium cannot access the majority of imide-dioxime sorption sites because of their saturation with vanadium. However, it is important to note that in the aforementioned studies the uranyl binding environment was not altered by the presence of vanadium, suggesting distinct binding sites may exist. Moreover, subsequent work has suggested the majority of the adsorbent graft chain is composed of the cyclic imidedioxime species (Das, Brown et al. 2016), in which case a definitive spectrum indicative of cyclic imidedioxime-bound uranyl would be expected, but was not consistent with the observed data. Equally important is the observation that brine-contacted adsorbents do not display the same binding environment as seawater-deployed adsorbents for either vanadium or uranium. Additional focused studies are necessary to conclusively determine whether uranium and vanadium truly compete for the same binding sites on polyamidoxime adsorbents. 


\section{REFERENCES}

Abney, C. W., S. Das, R. T. Mayes, L.-J. Kuo, J. Wood, G. Gill, M. Piechowicz, Z. Lin, W. Lin and S. Dai (2016). "A Report of Emergent Uranyl Binding Phenomena by an Amidoxime Phosphonic Acid CoPolymer." Physical Chemistry Chemical Physics 18(34): 23462-23468.

Abney, C. W., R. T. Mayes, M. Piechowicz, Z. Lin, V. Bryantsev, G. M. Veith, S. Dai and W. Lin (2016). "XAFS Investigation of Polyamidoxime-Bound Uranyl Contests the Paradigm from Small Molecule Studies." Energy \& Environmental Science 9(2): 448-453.

Astheimer, L., H. J. Schenk, E. G. Witte and K. Schwochau (1983). "Development of Sorbers for the Recovery of Uranium from Seawater. Part 2. The Accumulation of Uranium from Seawater by Resins Containing Amidoxime and Imidoxime Functional Groups." Separation Science and Technology 18(4): 307-339.

Baes, C. F., Jr. and R. E. Mesmer (1976). The Hydrolysis of Cations. New York, John Wiley \& Sons. Brown, S., Y. Yue, L.-J. Kuo, N. Mehio, M. Li, G. Gill, C. Tsouris, R. T. Mayes, T. Saito and S. Dai (2016). "Uranium Adsorbent Fibers Prepared by Atom-Transfer Radical Polymerization (ATRP) from Poly(vinyl chloride)-co-chlorinated Poly(vinyl chloride) (PVC-co-CPVC) Fiber." Industrial \& Engineering Chemistry Research 55(15): 4139-4148.

Calvin, S. (2013). XAFS for Everyone. Boca Raton, FL, CRC Press.

Das, S., S. Brown, R. T. Mayes, C. J. Janke, C. Tsouris, L. J. Kuo, G. Gill and S. Dai (2016). "Novel Poly(Imide Dioxime) Sorbents: Development and Testing for Enhanced Extraction of Uranium from Natural Seawater." Chemical Engineering Journal 298: 125-135.

Das, S., Y. Oyola, R. T. Mayes, C. J. Janke, L. J. Kuo, G. Gill, J. R. Wood and S. Dai (2016a). "Extracting Uranium from Seawater: Promising AF Series Adsorbents." Industrial \& Engineering Chemistry Research 55(15): 4110-4117.

Das, S., Y. Oyola, R. T. Mayes, C. J. Janke, L. J. Kuo, G. Gill, J. R. Wood and S. Dai (2016b). "Extracting Uranium from Seawater: Promising AI Series Adsorbents." Industrial \& Engineering Chemistry Research 55(15): 4103-4109.

Dobrynin, A. V. and M. Rubinstein (2005). "Theory of Polyelectrolytes in Solutions and at Surfaces." Progress in Polymer Science 30(11): 1049-1118.

Eloy, F. and R. Lenaers (1962). "The Chemistry of Amidoximes and Related Compounds." Chemical Reviews 62(2): 155-183.

Endrizzi, F., A. Melchior, M. Tolazzi and L. Rao (2015). "Complexation of Uranium(VI) with Glutarimidoxioxime: Thermodynamic and Computational Studies." Dalton Transactions 44(31): 1383513844.

Flicker Byers, M. and E. Schneider (2015). "Optimization of the Passive Recovery of Uranium from Seawater." Industrial \& Engineering Chemistry Research 55(15): 4351-4361.

Gill, G. A., L.-J. Kuo, C. J. Janke, J. Park, R. Jeters, G. Bonheyo, H.-B. Pan, C. M. Wai, T. Khangaonkar, L. Bianucci, J. Wood, M. G. Warner, S. Peterson, D. G. Abrecht, R. T. Mayes, C. Tsouris, Y. Oyola, J. E. Strivens, N. J. Schlafer, R. S. Addleman, W. Chouyyok, S. Das, J. Kim, K. O. Buesseler, C. F. Breier and E. D'Alessandro (2016). "The Uranium from Seawater Program at PNNL: Overview of Marine Testing, Adsorbent Characterization, Adsorbent Durability, Adsorbent Toxicity, and Deployment Studies." Industrial \& Engineering Chemistry Research 55(15): 4264-4277.

Ivanov, A. S. and V. S. Bryantsev (2016). "Assessing ligand selectivity for uranium over vanadium ions to aid in the discovery of superior adsorbents for extraction of UO22+ from seawater." Dalton Transactions 45(26): 10744-10751.

Kang, S. O., S. Vukovic, R. Custelcean and B. P. Hay (2012). "Cyclic Imide Dioximes: Formation and Hydrolytic Stability." Industrial \& Engineering Chemistry Research 51(19): 6619-6624.

Katritzky, A. R., L. Huang, M. Chahar, R. Sakhuja and C. D. Hall (2012). "The Chemistry of NHydroxyamidoximes, N-Aminoamidoximes, and Hydrazidines." Chemical Reviews 112(3): 1633-1649. 
Kim, J., C. Tsouris, R. T. Mayes, Y. Oyola, T. Saito, C. J. Janke, S. Dai, E. Schneider and D. Sachde (2013). "Recovery of Uranium from Seawater: A Review of Current Status and Future Research Needs." Separation Science and Technology 48(3): 367-387.

Kropf, A. J., J. Katsoudas, S. Chattopadhyay, T. Shibata, E. A. Lang, V. N. Zyryanov, B. Ravel, K. McIvor, K. M. Kemner, K. G. Scheckel, S. R. Bare, J. Terry, S. D. Kelly, B. A. Bunker and C. U. Segre (2010). "The new MRCAT (Sector 10) Bending Magnet beamline at the Advanced Photon Source." AIP Conference Proceedings 1234: 299-302.

Leggett, C. J., B. F. Parker, S. J. Teat, Z. Zhang, P. D. Dau, W. W. Lukens, S. M. Peterson, A. J. P. Cardenas, M. G. Warner, J. K. Gibson, J. Arnold and L. Rao (2016). "Structural and spectroscopic studies of a rare non-oxido V(v) complex crystallized from aqueous solution." Chemical Science 7(4): 27752786.

Lindner, H. and E. Schneider (2015). "Review of Cost Estimates for Uranium Recovery from Seawater." Energy Economics 49(0): 9-22.

Mehio, N., A. S. Ivanov, A. P. Ladshaw, S. Dai and V. S. Bryantsev (2016). "Theoretical Study of Oxovanadium(IV) Complexation with Formamidoximate: Implications for the Design of UranylSelective Adsorbents." Industrial \& Engineering Chemistry Research 55(15): 4231-4240.

Mehio, N., J. C. Johnson, S. Dai and V. S. Bryantsev (2015). "Theoretical study of the coordination behavior of formate and formamidoximate with dioxovanadium(v) cation: implications for selectivity towards uranyl." Physical Chemistry Chemical Physics 17(47): 31715-31726.

Morel, F. M. M. and N. M. Price (2003). "The Biogeochemical Cycles of Trace Metals in the Oceans." Science 300(5621): 944-947.

Pan, H.-B., L.-J. Kuo, C. M. Wai, N. Miyamoto, R. Joshi, J. Wood, J. E. Strivens, C. J. Janke, Y. Oyola, S. Das, R. T. Mayes and G. A. Gill (2015). "Elution of Uranium and Transition Metals from AmidoximeBased Polymer Adsorbents for Sequestering Uranium from Seawater." Industrial \& Engineering Chemistry Research.

Ravel, B. and M. Newville (2005). "Athena, Artemis, Hephaestus: Data Analysis for X-ray Absorption Spectroscopy using IFEFFIT." Journal of Synchrotron Radiation 12(4): 537-541.

Rehr, J. J. and R. C. Albers (2000). "Theoretical Approaches to X-ray Absorption Fine Structure." Reviews of Modern Physics 72(3): 621-654.

Rehr, J. J., J. J. Kas, F. D. Vila, M. P. Prange and K. Jorissen (2010). "Parameter-free calculations of Xray spectra with FEFF9." Physical Chemistry Chemical Physics 12(21): 5503-5513.

Saito, T., S. Brown, S. Chatterjee, J. Kim, C. Tsouris, R. T. Mayes, L.-J. Kuo, G. Gill, Y. Oyola, C. J. Janke and S. Dai (2014). "Uranium Recovery from Seawater: Development of Fiber Adsorbents Prepared via Atom-Transfer Radical Polymerization." Journal of Materials Chemistry A 2(35): 14674-14681. Tamada, M. (2010). Current Status of Technology for Collection of Uranium from Seawater. Toh Tuck Link, Singapore, World Scientific Publishing Co. Pte. Ltd.

Tian, G., S. Teat and L. Rao (2013). "Thermodynamic Studies of U(VI) Complexation with Glutardiamidoxime for Sequestration of Uranium from Seawater." Dalton Transactions 42(16): 56905696.

Tian, G., S. Teat, Z. Zhang and L. Rao (2012). "Sequestering Uranium from Seawater: Binding Strength and Modes of Uranyl Complexes with Glutarimidedioxime." Dalton Transactions 41(38): 11579-11586. Vukovic, S., L. A. Watson, S. O. Kang, R. Custelcean and B. P. Hay (2012). "How Amidoximate Binds the Uranyl Cation." Inorganic Chemistry 51(6): 3855-3859. 